\title{
The Dirac Matrix Group and Fierz Transformations
}

\author{
E. DE VRIES and A. J. VAN ZANTEN \\ Institute for Theoretical Physics, University of Groningen, The Netherlands
}

Received November 26, 1969; in revised form March 11, 1970

\begin{abstract}
The representation theory of the group generated by the Dirac matrices is studied. It is shown that the Fierz transformation can be expressed in terms of Racah coefficients of this group. A number of generalized Fierz transformations have been found. Simple rules are given for calculating Fierz invariants and anti-invariants.
\end{abstract}

\section{Introduction}

When performing explicit calculations on four-fermion interactions it is often expedient to apply the so-called Fierz transformation [1-3] and to make use of the Fierz invariants and anti-invariants [4-7]. The usual derivation of the explicit form of this transformation does not provide much insight in the underlying principles and is somewhat clumsy. Case [8] studied the Fierz identities in relation with the theory of spinor representations of orthogonal groups. He found for every orthogonal group the corresponding Fierz transformation using methods which do not differ essentially from the conventional procedure.

In a certain sense the Fierz transformation resembles crossing relations. Now it is well-known that the crossing matrix for isospin can be expressed as a Racah coefficient of the relevant group $S U(2)$ [9-12]. In this paper we study the group generated by the Dirac matrices and apply the well-known techniques of $S U(2)$, embodying elements such as Clebsch-Gordan coefficients (or Wigner coefficients), Racah coefficients, irreducible tensor operators and Wigner-Eckart theorem. This is possible, because this group is simply reducible. A crucial point will be the interpretation of the $\Gamma$-matrices as tensor operators. We show that the Fierz matrix is related to the Racah coefficients of the Dirac matrix group. Furthermore several other generalized Fierz transformations are given most of which do not seem to have been known before. The method with which we obtain these results gives more insight in the nature of the Fierz transformations and provides us with simple rules to calculate all kinds of Fierz matrices and the corresponding invariants and anti-invariants. 
The above mentioned techniques can be applied to all finite groups related to the spin representations of orthogonal and pseudo-orthogonal groups $[13,14]$. We shall call them Clifford groups. The quaternion group is such a group and because it can be manipulated much easier we shall often use this group as an illustration.

\section{Dirac Matrix Group and Quaternion Group}

a) Definition and General Properties

The Dirac matrix group is generated by the usual Dirac matrices $\gamma^{0}, \gamma^{1}, \gamma^{2}$ and $\gamma^{3}$, which obey

$$
\gamma^{\mu} \gamma^{v}+\gamma^{v} \gamma^{\mu}=2 g^{\mu v} \quad(\mu, v=0,1,2,3),
$$

with $g^{\mu \mu}=(1,-1,-1,-1)$.

We introduce $\gamma^{5}=\gamma^{0} \gamma^{1} \gamma^{2} \gamma^{3}$, for which we have $\left(\gamma^{5}\right)^{2}=-1$ and $\gamma^{\mu} \gamma^{5}+\gamma^{5} \gamma^{\mu}=0$.

Hence

$$
\gamma^{a} \gamma^{b}+\gamma^{b} \gamma^{a}=2 g^{a b} \quad(a, b=0,1,2,3,5),
$$

with $g^{a a}=(1,-1,-1,-1,-1)$.

The group elements are then $\pm 1, \pm \gamma^{a}, \pm \gamma^{a} \gamma^{b}(a<b)$.

It can be easily verified that this group has 32 elements which, when no confusion arises, will be denoted by $\pm \Gamma_{i}(i=1,2, \ldots, 16)$.

The group has seventeen classes: (1), $(-1),\left(\gamma^{a},-\gamma^{a}\right)$ and $\left(\gamma^{a} \gamma^{b},-\gamma^{a} \gamma^{b}\right)$. There exists one faithful, four-dimensional irreducible representation, whereas the sixteen remaining irreducible representations are all onedimensional. The one-dimensional irreducible representations will be denoted by $\left(1_{p}\right)(p=1, \ldots, 16)$ and the four-dimensional irreducible representation by (4). (The symbol $\left(1_{1}\right)$ stands for the trivial representation.)

A unitary form of the four-dimensional representation is generated by

$$
\begin{aligned}
D^{(4)}\left(\gamma^{0}\right) & =\left[\begin{array}{rrrr}
1 & 0 & 0 & 0 \\
0 & 1 & 0 & 0 \\
0 & 0 & -1 & 0 \\
0 & 0 & 0 & -1
\end{array}\right], D^{(4)}\left(\gamma^{1}\right)=\left[\begin{array}{rrrr}
0 & 0 & 0 & 1 \\
0 & 0 & 1 & 0 \\
0 & -1 & 0 & 0 \\
-1 & 0 & 0 & 0
\end{array}\right], \\
D^{(4)}\left(\gamma^{2}\right) & =\left[\begin{array}{rrrr}
0 & 0 & 0 & -i \\
0 & 0 & i & 0 \\
0 & i & 0 & 0 \\
-i & 0 & 0 & 0
\end{array}\right], D^{(4)}\left(\gamma^{3}\right)=\left[\begin{array}{rrrr}
0 & 0 & 1 & 0 \\
0 & 0 & 0 & -1 \\
-1 & 0 & 0 & 0 \\
0 & 1 & 0 & 0
\end{array}\right] .
\end{aligned}
$$


By applying formula (5-84) of Ref. [15] it can be shown that all onedimensional representations are integer representations and the fourdimensional irreducible representation is half-integer. With the help of the character table we can reduce the Kronecker product of two arbitrary irreducible representations. The results are

$$
\begin{aligned}
\left(1_{p}\right) \otimes\left(1_{q}\right) & =\left(1_{r}\right), \\
\left(1_{p}\right) \otimes(4) & =(4), \\
(4) \otimes(4) & =\sum_{p=1}^{16}\left(1_{p}\right) .
\end{aligned}
$$

These are the Clebsch-Gordan series of the Dirac matrix group. We see that in a Kronecker product of two irreducible representations a certain irreducible representation occurs once or not at all. As furthermore all characters of the group are real we have to do with a so-called simply reducible group $[15,16]$.

As already announced in our introduction we shall often use the quaternion group as an illustration. Therefore we give here also the similar properties of this group. The elements of the quaternion group are $\pm 1, \pm i, \pm j$ and $\pm k$, with the multiplication rules $i^{2}=j^{2}=k^{2}=-1$ and $i j=k, j k=i$ and $k i=j$. (So $i j=-j i$ etc.) Alternatively the eight elements will be denoted by $\pm \Sigma_{1}= \pm 1, \pm \Sigma_{2}= \pm i, \pm \Sigma_{3}= \pm j$ and $\pm \Sigma_{4}= \pm k$. This group has five classes $(1),(-1),(i,-i),(j,-j)$ and $(k,-k)$. There exists one faithful, two-dimensional irreducible representation

$$
\Sigma_{1}=\left(\begin{array}{ll}
1 & 0 \\
0 & 1
\end{array}\right), \quad \Sigma_{2}=\left(\begin{array}{ll}
0 & i \\
i & 0
\end{array}\right), \quad \Sigma_{3}=\left(\begin{array}{rr}
0 & -1 \\
1 & 0
\end{array}\right), \quad \Sigma_{4}=\left(\begin{array}{rr}
i & 0 \\
0 & -i
\end{array}\right) .
$$

\begin{tabular}{|c|c|c|c|c|c|c|}
\hline & \multicolumn{5}{|c|}{$\rightarrow$ irreducible representation } \\
\hline & & $1_{1}$ & $1_{2}$ & $1_{3}$ & $1_{4}$ & 2 \\
\hline class & 1 & 1 & 1 & 1 & 1 & 2 \\
\hline$\downarrow$ & -1 & 1 & 1 & 1 & 1 & -2 \\
\hline & $(i,-i)$ & 1 & 1 & -1 & -1 & 0 \\
\hline & $(j,-j)$ & 1 & -1 & 1 & -1 & 0 \\
\hline & $(k,-k)$ & 1 & -1 & -1 & 1 & 0 \\
\hline
\end{tabular}

The four remaining irreducible representations are one-dimensional. The character table of the quaternion group is as follows

Here, just as in the case of the Dirac matrix group all one-dimensional representations are integer representations, whereas the two-dimensional irreducible representation is half-integer. 
The Clebsch-Gordan series are

$$
\begin{aligned}
\left(1_{p}\right) \otimes\left(1_{q}\right) & =\left(1_{r}\right), \\
\left(1_{p}\right) \otimes(2) & =(2), \\
(2) \otimes(2) & =\sum_{p=1}^{4}\left(1_{p}\right) .
\end{aligned}
$$

Once again the quaternion group is simply reducible.

\section{b) Wigner Coefficients and 6j-Symbols}

To reduce explicitly the Kronecker product of two irreducible representations we need Wigner coefficients (Clebsch-Gordan coefficients). Because we have to do with simply reducible groups we can apply formula (5-149) of Ref. [15] with $\kappa_{1}=\lambda_{1}, \kappa_{2}=\lambda_{2}$ and $\kappa_{3}=\lambda_{3}$ to calculate the absolute value of a Wigner coefficient. For fixed $j_{1}, j_{2}$ and $j_{3}$ the phase can be chosen for one arbitrary Wigner coefficient. Then the phases of the other coefficients with the same $j_{1}, j_{2}$ and $j_{3}$ can be found by means of formula (5-149). In the case of the quaternion group the nontrivial Wigner coefficients $\left(\begin{array}{lll}2 & 2 & 1 \\ m_{1} & m_{2} & 1\end{array}\right)$ are given in the following table

\begin{tabular}{llllll}
\hline$\rightarrow$ & $1_{p}$ & $1_{1}$ & $1_{4}$ & $1_{2}$ & $1_{3}$ \\
$\downarrow m_{1}$ & $m_{2}$ & & & & \\
\hline
\end{tabular}

\begin{tabular}{cccccc}
1 & 2 & $+1 / \sqrt{2}$ & $+1 / \sqrt{2}$ & 0 & 0 \\
2 & 1 & $-1 / \sqrt{2}$ & $+1 / \sqrt{2}$ & 0 & 0 \\
1 & 1 & 0 & 0 & $+1 / \sqrt{2}$ & $+1 / \sqrt{2}$ \\
2 & 2 & 0 & 0 & $-1 / \sqrt{2}$ & $+1 / \sqrt{2}$ \\
\hline
\end{tabular}

The matrix which transforms the given two-dimensional irreducible representation into its complex conjugate form is

$$
U_{\kappa \lambda}=\left(\begin{array}{cc}
2 \\
\kappa & \lambda
\end{array}\right)=\sqrt{2}\left(\begin{array}{lll}
2 & 1 & 2 \\
\kappa & 1 & \lambda
\end{array}\right)=\sqrt{2}\left(\begin{array}{lll}
2 & 2 & 1 \\
\lambda & \kappa & 1
\end{array}\right)=\left[\begin{array}{rr}
0 & -1 \\
1 & 0
\end{array}\right] .
$$

(See Ref. [15], formula (5-153)).

Now the $1 j m$-symbols $\left(\begin{array}{cc}1 & \\ 1 & 1\end{array}\right)$ are also fixed and can easily be calculated with formula (11a) of Ref. [16]:

$$
\left(\begin{array}{lll}
j_{1} & j_{2} & j_{3} \\
v_{1} & v_{2} & v_{3}
\end{array}\right)^{*}=\left(\begin{array}{ccc}
j_{1} & j_{2} & j_{3} \\
\lambda_{1} & \lambda_{2} & \lambda_{3}
\end{array}\right)\left(\begin{array}{cc}
j_{1} \\
\lambda_{1} & v_{1}
\end{array}\right)^{*}\left(\begin{array}{cc}
j_{2} \\
\lambda_{2} & v_{2}
\end{array}\right)^{*}\left(\begin{array}{cc}
j_{3} \\
\lambda_{3} & v_{3}
\end{array}\right)^{*}
$$


Put $j_{1}=j_{2}=2$ and $j_{3}=1_{p}$ and take $v_{1}, v_{2}$ and $v_{3}$ such that

The results are

$$
\left(\begin{array}{lll}
2 & 2 & 1_{p} \\
v_{1} & v_{2} & v_{3}
\end{array}\right) \neq 0
$$

$$
\left(\begin{array}{cc}
1_{1} \\
1 & 1
\end{array}\right)=+1, \quad\left(\begin{array}{cc}
1_{2} \\
1 & 1
\end{array}\right)=-1, \quad\left(\begin{array}{cc}
1_{3} \\
1 & 1
\end{array}\right)=+1, \quad\left(\begin{array}{cc}
1_{4} \\
1 & 1
\end{array}\right)=-1 .
$$

The $6 j$-symbols which turn out to be of interest in the following sections have the form $\left\{\begin{array}{lll}2 & 2 & 1_{p} \\ 2 & 2 & 1_{q}\end{array}\right\}$. These can be calculated with formula (27a) of Ref. [16]. One finds

\begin{tabular}{lrrrr}
\hline $\overrightarrow{1_{p}}$ & $1_{1}$ & $1_{2}$ & $1_{3}$ & $1_{4}$ \\
$\downarrow 1_{q}$ & & & & \\
\hline $1_{1}$ & $-\frac{1}{2}$ & $\frac{1}{2}$ & $\frac{1}{2}$ & $\frac{1}{2}$ \\
$1_{2}$ & $\frac{1}{2}$ & $-\frac{1}{2}$ & $\frac{1}{2}$ & $\frac{1}{2}$ \\
$1_{3}$ & $\frac{1}{2}$ & $\frac{1}{2}$ & $-\frac{1}{2}$ & $\frac{1}{2}$ \\
$1_{4}$ & $\frac{1}{2}$ & $\frac{1}{2}$ & $\frac{1}{2}$ & $-\frac{1}{2}$ \\
\hline
\end{tabular}

The Wigner coefficients, the $6 j$-symbols and the $1 j m$-symbols for the Dirac matrix group can be calculated in the same way.

\section{c) Irreducible Tensor Operators and Wigner-Eckart Theorem}

When studying the rotation group, $S U(2)$ or $S U(3)$ the irreducible tensor operators play an important role. A set of operators $T_{m}^{j}$ are said to be the components of an irreducible tensor operator of $\operatorname{rank} j$ if they satisfy

$$
R T_{m}^{j} R^{-1}=\sum_{m^{\prime}} D_{m^{\prime} m}^{(j)}(R) T_{m^{\prime}}^{j} .
$$

Here, $D_{m^{\prime} m}^{(j)}(R)$ is the matrix of the element $R$ in the irreducible representation $(j)$. Intuitively one finds that in the case of the quaternion group the group elements $\Sigma_{i}$ can also be interpreted as irreducible tensor operators belonging to the one-dimensional representations of the group. For instance, $\Sigma_{2}$ belongs to the irreducible representation which we labelled by $\left(1_{2}\right)$ :

$$
\begin{aligned}
& \Sigma_{1} \Sigma_{2}\left(\Sigma_{1}\right)^{-1}=+\Sigma_{2}, \\
& \Sigma_{2} \Sigma_{2}\left(\Sigma_{2}\right)^{-1}=+\Sigma_{2}, \\
& \Sigma_{3} \Sigma_{2}\left(\Sigma_{3}\right)^{-1}=-\Sigma_{2}, \\
& \Sigma_{4} \Sigma_{2}\left(\Sigma_{4}\right)^{-1}=-\Sigma_{2} .
\end{aligned}
$$


The correspondence between the $\Sigma_{i}$ considered as tensor operators and the one-dimensional representations is as follows

$$
\Sigma_{1} \leftrightarrow\left(1_{1}\right), \quad \Sigma_{2} \leftrightarrow\left(1_{2}\right), \quad \Sigma_{3} \leftrightarrow\left(1_{3}\right), \quad \Sigma_{4} \leftrightarrow\left(1_{4}\right) .
$$

Loosely speaking the one-dimensional representations can be characterized by a group element, which provides a kind of "natural" labelling. An analogous correspondence for the Dirac matrix group can be given.

A more rigorous treatment of irreducible tensor operators of finite groups will be postponed to a forthcoming publication.

For matrix elements of irreducible tensors belonging to a simply reducible group the Wigner-Eckart theorem holds (see formula (67) of Ref. [16]). In our notation we have

$$
\left\langle j m\left|T_{M}^{J}\right| j^{\prime} m^{\prime}\right\rangle=\sum_{\mu}\left(\begin{array}{lll}
j & J & j^{\prime} \\
\mu & M & m^{\prime}
\end{array}\right)\left(\begin{array}{cc}
j \\
\mu & m
\end{array}\right)^{*}\left\langle j\left\|T^{J}\right\| j^{\prime}\right\rangle .
$$

Here, $\left\langle j\left\|T^{J}\right\| j^{\prime}\right\rangle$ is the so-called reduced matrix element. Wigner wrote this as

$$
\left\langle j m\left|T_{M}^{J}\right| j^{\prime} m^{\prime}\right\rangle=\left(\begin{array}{lll}
m & J & j^{\prime} \\
j & M & m^{\prime}
\end{array}\right)\left\langle j\left\|T^{J}\right\| j^{\prime}\right\rangle,
$$

with the convention

$$
\left(\begin{array}{lll}
m & J & j^{\prime} \\
j & M & m^{\prime}
\end{array}\right)=\sum_{\mu}\left(\begin{array}{lll}
j & J & j^{\prime} \\
\mu & M & m^{\prime}
\end{array}\right)\left(\begin{array}{cc}
j \\
\mu & m
\end{array}\right)^{*} .
$$

By means of these relations the reduced matrix elements $\left\langle 2\left\|\Sigma_{i}\right\| 2\right\rangle$ can easily be calculated and we find

$$
\begin{aligned}
& \left\langle 2\left\|\Sigma_{1}\right\| 2\right\rangle=+\sqrt{2}, \\
& \left\langle 2\left\|\Sigma_{2}\right\| 2\right\rangle=-i \sqrt{2}, \\
& \left\langle 2\left\|\Sigma_{3}\right\| 2\right\rangle=-\sqrt{2}, \\
& \left\langle 2\left\|\Sigma_{4}\right\| 2\right\rangle=+i \sqrt{2} .
\end{aligned}
$$

The question arises what the irreducible tensor operator corresponding to the two-dimensional irreducible representation of the quaternion group looks like. When we have found this tensor operator, all possibilities for irreducible tensor operators are exhausted, because the quaternion group does not have any other irreducible representation. The Wigner-Eckart theorem allows us to construct an explicit matrix representation of this tensor operator by applying formula $(2 b)$ and using the Wigner coefficients given in the table in Section $2 \mathrm{~b}$. 
Calculations show that the general form of the tensor operator of rank 2 is

$$
\begin{aligned}
& T_{1}^{2}=\left[\begin{array}{rrrrrr}
0 & 0 & 0 & 0 & 0 & \xi_{1} \\
0 & 0 & 0 & 0 & -\xi_{2} & 0 \\
0 & 0 & 0 & 0 & \xi_{3} & 0 \\
0 & 0 & 0 & 0 & 0 & -\xi_{4} \\
-\xi_{5} & 0 & 0 & \xi_{8} & 0 & 0 \\
0 & -\xi_{6} & -\xi_{7} & 0 & 0 & 0
\end{array}\right], \\
& T_{2}^{2}=\left[\begin{array}{rrrrrr}
0 & 0 & 0 & 0 & -\xi_{1} & 0 \\
0 & 0 & 0 & 0 & 0 & \xi_{2} \\
0 & 0 & 0 & 0 & 0 & \xi_{3} \\
0 & 0 & 0 & 0 & -\xi_{4} & 0 \\
0 & -\xi_{6} & \xi_{7} & 0 & 0 & 0 \\
-\xi_{5} & 0 & 0 & -\xi_{8} & 0 & 0
\end{array}\right] .
\end{aligned}
$$

Here the $\xi_{i}$ are, up to factors, the reduced matrix elements $\left\langle j\left\|T^{2}\right\| j^{\prime}\right\rangle$. The above matrix representation is in the reducible vector space of the direct sum $\left(1_{1}\right) \oplus\left(1_{2}\right) \oplus\left(1_{3}\right) \oplus\left(1_{4}\right) \oplus(2)$ (the rows and columns are numbered with $(j, m)=\left(1_{1}, 1\right),\left(1_{2}, 1\right),\left(1_{3}, 1\right),\left(1_{4}, 1\right),(2,1)$ and $\left.(2,2)\right)$.

In this case an explicit matrix representation for $R$ in formula (1) is given by

$$
R\left(\Sigma_{i}\right)=D^{\left(1_{1}\right)}\left(\Sigma_{i}\right) \oplus D^{\left(1_{2}\right)}\left(\Sigma_{i}\right) \oplus D^{\left(1_{3}\right)}\left(\Sigma_{i}\right) \oplus D^{\left(1_{4}\right)}\left(\Sigma_{i}\right) \oplus D^{(2)}\left(\Sigma_{i}\right) .
$$

Analogous calculations can be done for the Dirac matrix group.

\section{Fierz Transformations}

\section{a) Field Theoretic Definition}

In field theory one defines in the following way a transformation of coupling constants. The interaction hamiltonians

and

$$
H=\sum_{I=1}^{5} g_{I}\left(\bar{\psi}_{a} \Gamma_{I, \alpha} \psi_{b}\right)\left(\bar{\psi}_{c} \Gamma_{I}^{\alpha} \psi_{d}\right)
$$

$$
H=\sum_{I=1}^{5} g_{I}^{\prime}\left(\bar{\psi}_{a} \Gamma_{I, \alpha} \psi_{d}\right)\left(\bar{\psi}_{c} \Gamma_{I}^{\alpha} \psi_{b}\right)
$$

(with summation convention for $\alpha$ ) are equivalent, if there is a relationship $g_{I}^{\prime}=\sum_{K} L_{I K} g_{K}$ among the coupling
constants $g_{I}$ and $g_{I}^{\prime}$. 
The $\Gamma_{I, \alpha} \Gamma_{I}^{\alpha}$ are Lorentz invariant combinations of $\Gamma$-matrices as defined e.g. in Refs. [1] and [2]. (When it does not give rise to confusion, here and in the following sections we shall simply use $\Gamma_{i}$ to denote the four-dimensional irreducible representation $D^{(4)}\left(\Gamma_{i}\right)$.) A trivial property of the matrix $L$ is $L^{2}=1$. It can be understood easily, that an equivalent definition is given by

$$
\left(\bar{\psi}_{a} \Gamma_{I, \alpha} \psi_{b}\right)\left(\bar{\psi}_{c} \Gamma_{I}^{\alpha} \psi_{d}\right)=\sum_{K=1}^{5} F_{K I}\left(\bar{\psi}_{a} \Gamma_{K, \alpha} \psi_{d}\right)\left(\bar{\psi}_{c} \Gamma_{K}^{\alpha} \psi_{b}\right)
$$

The $F$-matrix is the transposed $L$-matrix and has the form

$$
F_{I K}=\frac{1}{4}\left[\begin{array}{rrrrr}
1 & 1 & 1 & 1 & 1 \\
4 & -2 & 0 & 2 & -4 \\
6 & 0 & -2 & 0 & 6 \\
4 & 2 & 0 & -2 & -4 \\
1 & -1 & 1 & -1 & 1
\end{array}\right]
$$

We now want to study this transformation with the methods developed in group theory and the techniques referred to in Section 2.

In order to cast the problem in a more appropriate form we define an "elementary" Fierz transformation

$$
\left(\bar{\psi}_{a} \Gamma_{i} \psi_{b}\right)\left(\bar{\psi}_{c} \Gamma_{i} \psi_{d}\right)=\sum_{k=1}^{16} F_{k i}\left(\bar{\psi}_{a} \Gamma_{k} \psi_{d}\right)\left(\bar{\psi}_{c} \Gamma_{k} \psi_{b}\right)
$$

Here the Lorentz structure has been dropped and the summation runs over the sixteen elements $1, \gamma^{a}$ and $\gamma^{a} \gamma^{b}(a<b)$. We may confine ourselves to these group elements, because $+\Gamma_{i}$ gives the same contribution as $-\Gamma_{i}$ in the above formula.

With the aid of the properties $\operatorname{Tr} \Gamma_{i}=0$ for all $\Gamma_{i} \neq 1$ and $\Gamma_{i}^{-1}= \pm \Gamma_{i}$ it can be shown that

$$
F_{i k}=\frac{1}{16} \operatorname{Tr}\left(\Gamma_{i} \Gamma_{k}^{-1} \Gamma_{i} \Gamma_{k}^{-1}\right)=\frac{1}{16} \operatorname{Tr}\left(\Gamma_{i} \Gamma_{k} \Gamma_{i} \Gamma_{k}\right) .
$$

It is obvious that $F$ is a hermitian and also a unitary operator

$$
F=F^{+}=F^{-1} .
$$

As the quaternion group has similar properties as the Dirac matrix group $\left(\operatorname{Tr} \Sigma_{i}=0\right.$ for all $\Sigma_{i} \neq 1$ and $\left.\Sigma_{i}^{-1}= \pm \Sigma_{i}\right)$ an analogous transformation can be defined in this case

$$
\left(\bar{\psi}_{a} \Sigma_{i} \psi_{b}\right)\left(\bar{\psi}_{c} \Sigma_{i} \psi_{d}\right)=\sum_{k=1}^{4} F_{k i}\left(\bar{\psi}_{a} \Sigma_{k} \psi_{d}\right)\left(\bar{\psi}_{c} \Sigma_{k} \psi_{b}\right),
$$


with

$$
F_{i k}=\frac{1}{4} \operatorname{Tr}\left(\Sigma_{i} \Sigma_{k} \Sigma_{i} \Sigma_{k}\right) .
$$

Explicit calculation gives

$$
F=\frac{1}{2}\left[\begin{array}{rrrr}
1 & -1 & -1 & -1 \\
-1 & 1 & -1 & -1 \\
-1 & -1 & 1 & -1 \\
-1 & -1 & -1 & 1
\end{array}\right]
$$

b) Invariants of the Elementary Fierz Transformation

A Fierz invariant (c.q. anti-invariant) is a form

$$
\sum_{i} x_{i}\left(\bar{\psi}_{a} \Gamma_{i} \psi_{b}\right)\left(\bar{\psi}_{c} \Gamma_{i} \psi_{d}\right)
$$

which does not change (c.q. changes sign) under a Fierz transformation. In general we have

$$
\sum_{i} x_{i}\left(\bar{\psi}_{a} \Gamma_{i} \psi_{b}\right)\left(\bar{\psi}_{c} \Gamma_{i} \psi_{d}\right)=\sum_{i, k} x_{i} F_{k i}\left(\bar{\psi}_{a} \Gamma_{k} \psi_{d}\right)\left(\bar{\psi}_{c} \Gamma_{k} \psi_{b}\right)
$$

If we want this to be an invariant (c.q. anti-invariant) we shall have to require

$$
\sum_{i} x_{i}\left(\bar{\psi}_{a} \Gamma_{i} \psi_{b}\right)\left(\bar{\psi}_{c} \Gamma_{i} \psi_{d}\right)=\lambda \sum_{k} x_{k}\left(\bar{\psi}_{a} \Gamma_{k} \psi_{d}\right)\left(\bar{\psi}_{c} \Gamma_{k} \psi_{b}\right),
$$

with $\lambda=+1$ for an invariant and $\lambda=-1$ for an anti-invariant. Therefore the coefficients $x_{i}$ have to satisfy $\sum_{i} F_{k i} x_{i}=\lambda x_{k}$, i. e. the $x_{i}$ are the components of the eigenvectors of the matrix $F$ whereas the $\lambda$ 's are the corresponding eigenvalues. Because $F$ is a hermitian matrix, whose square equals 1 , all eigenvalues are +1 or -1 and all eigenvectors exist.

Theorem. The Fierz invariants and anti-invariants are given by $\sum_{i} \chi_{i}^{\left(1_{p}\right)}\left(\bar{\psi}_{a} \Gamma_{i} \psi_{b}\right)\left(\bar{\psi}_{c} \Gamma_{i} \psi_{d}\right)$, where $\chi_{i}^{\left(i_{p}\right)}$ are the characters of the one-dimensional representations of the Dirac matrix group. Odd representations $\left(1_{p}\right)$ give rise to an invariant, even representations to an anti-invariant.

Proof. We shall interpret $\left(\bar{\psi}_{a} \Gamma_{i} \psi_{b}\right)$ in the following way. Up to now $\psi_{a}, \psi_{b}, \ldots$ have been arbitrary spinors. However, it is sufficient to prove the theorem for the case where $\psi_{a}, \psi_{b}, \ldots$ are basis spinors. Then we have

$$
\left(\bar{\psi}_{a} \Gamma_{i} \psi_{b}\right)=\left(\Gamma_{i}\right)_{a b}=D_{a b}^{(4)}\left(\Gamma_{i}\right) .
$$

The characters of the one-dimensional representations are the representations

$$
\chi_{i}^{\left(1_{p}\right)}=\chi^{\left(1_{p}\right)}\left(\Gamma_{i}\right)=D^{\left(1_{p}\right)}\left(\Gamma_{i}\right) .
$$


The expression

$$
\sum_{i=1}^{16} \chi_{i}^{\left(1_{p}\right)}\left(\bar{\psi}_{a} \Gamma_{i} \psi_{b}\right)\left(\bar{\psi}_{c} \Gamma_{i} \psi_{d}\right)
$$

becomes in the new notation

$$
\sum_{i=1}^{16} D^{\left(1_{p}\right)}\left(\Gamma_{i}\right) D_{a b}^{(4)}\left(\Gamma_{i}\right) D_{c d}^{(4)}\left(\Gamma_{i}\right)
$$

The summation may be extended over the whole group, because it is clear that $\Gamma_{i}$ and $-\Gamma_{i}$ give equal contributions to the sum. We then get

$$
\sum_{R \in G} D^{\left(1_{p}\right)}(R) D_{a b}^{(4)}(R) D_{c d}^{(4)}(R)
$$

We now apply formula (5-149) of Ref. [15] and a number of symmetry relations

$$
\begin{aligned}
\sum_{R \in G} D^{\left(1_{p}\right)}(R) D_{a b}^{(4)}(R) D_{c d}^{(4)}(R) & =g\left(\begin{array}{lll}
1_{p} & 4 & 4 \\
1 & a & c
\end{array}\right)^{*}\left(\begin{array}{lll}
1_{p} & 4 & 4 \\
1 & b & d
\end{array}\right) \\
& =g\left(\begin{array}{lll}
4 & 4 & 1_{p} \\
a & c & 1
\end{array}\right)^{*}\left(\begin{array}{lll}
4 & 4 & 1_{p} \\
b & d & 1
\end{array}\right) \\
& =\lambda g\left(\begin{array}{lll}
4 & 4 & 1 \\
a & c & 1
\end{array}\right)^{*}\left(\begin{array}{lll}
4 & 4 & 1_{p} \\
d & b & 1
\end{array}\right) \\
& =\lambda \sum_{R \in G} D^{\left(1_{p}\right)}(R) D_{a d}^{(4)}(R) D_{c b}^{(4)}(R),
\end{aligned}
$$

with $\lambda=-(-1)^{\left(1_{p}\right)}= \pm 1$, depending on the odd or even character of the one-dimensional representation $\left(1_{p}\right)$.

Hence in usual notation

$$
\sum_{i=1}^{16} \chi_{i}^{\left(1_{p}\right)}\left(\bar{\psi}_{a} \Gamma_{i} \psi_{b}\right)\left(\bar{\psi}_{c} \Gamma_{i} \psi_{d}\right)=\lambda \sum_{i=1}^{16} \chi_{i}^{(1 p)}\left(\bar{\psi}_{a} \Gamma_{i} \psi_{d}\right)\left(\bar{\psi}_{c} \Gamma_{i} \psi_{b}\right)
$$

\section{c) Elementary Fierz Matrix and 6j-Symbols}

The Fierz matrix $F_{i k}$ was defined by

$$
\left(\bar{\psi}_{a} \Gamma_{i} \psi_{b}\right)\left(\bar{\psi}_{c} \Gamma_{i} \psi_{d}\right)=\sum_{k=1}^{16} F_{k i}\left(\bar{\psi}_{a} \Gamma_{k} \psi_{d}\right)\left(\bar{\psi}_{c} \Gamma_{k} \psi_{b}\right)
$$

Once again we choose basis spinors for $\psi_{a}$, etc. Because these spinors belong to the four-dimensional irreducible representation we shall denote them by $|4, a\rangle=\psi_{a}$. In this section the $\Gamma_{i}$ will be considered as irreducible tensor operators of rank $\left(1_{i}\right)$ as explained in Section 2 c. Then 
the above definition takes the form

$$
\left\langle 4, a\left|\Gamma_{i}\right| 4, b\right\rangle\left\langle 4, c\left|\Gamma_{i}\right| 4, d\right\rangle=\sum_{k=1}^{16} F_{k i}\left\langle 4, a\left|\Gamma_{k}\right| 4, d\right\rangle\left\langle 4, c\left|\Gamma_{k}\right| 4, b\right\rangle .
$$

We shall need two lemma's to derive the relationship between the Fierz matrix $F_{k i}$ and the $6 j$-symbol $\left\{\begin{array}{lll}4 & 4 & 1_{i} \\ 4 & 4 & 1_{k}\end{array}\right\}$.

Lemma I. Let $\Gamma_{i}^{2}=\eta_{i} \cdot 1$ ( 1 is the $4 \times 4$-unit matrix), where $\eta_{i}= \pm 1$ and let $\Gamma^{i}$ transform as an irreducible tensor operator of rank $\left(1_{i}\right)$. Then we have

$$
\eta_{i}=(-1)^{\left(1_{2}\right)}
$$

Proof. In Section $3 \mathrm{~b}$ we proved the following relation

$$
\sum_{k=1}^{16} F_{i k} \chi_{k}^{\left(1_{j}\right)}=-(-1)^{\left(1_{j}\right)} \chi_{i}^{\left(1_{j}\right)}
$$

where $F_{i k}=\frac{1}{16} \operatorname{Tr}\left(\Gamma_{i} \Gamma_{k} \Gamma_{i} \Gamma_{k}\right)$.

Because $\Gamma_{i}$ is an irreducible tensor operator of rank $\left(1_{i}\right)$ we have

or

$$
\Gamma_{k} \Gamma_{i} \Gamma_{k}^{-1}=D^{\left(1_{1}\right)}\left(\Gamma_{k}\right) \Gamma_{i}=\chi^{\left(1_{i}\right)}\left(\Gamma_{k}\right) \Gamma_{i}=\chi_{k}^{\left(1_{1}\right)} \Gamma_{i},
$$

$$
\Gamma_{k} \Gamma_{i} \Gamma_{k}^{-1} \Gamma_{i}^{-1}=\chi_{k}^{\left(1_{1}\right)} \cdot 1 \text {. }
$$

On the other hand

hence

$$
\Gamma_{k} \Gamma_{i} \Gamma_{k}^{-1} \Gamma_{i}^{-1}=\eta_{i} \eta_{k} \Gamma_{k} \Gamma_{i} \Gamma_{k} \Gamma_{i},
$$

$$
F_{i k}=\frac{1}{4} \eta_{i} \eta_{k} \chi_{k}^{\left(1_{i}\right)} .
$$

If we substitute this expression in formula (17) we obtain

$$
\frac{1}{4} \eta_{i} \sum_{k=1}^{16} \eta_{k} \chi_{k}^{\left(1_{2}\right)} \chi_{k}^{(1, j)}=-(-1)^{\left(1_{j}\right)} \chi_{i}^{\left(1_{j}\right)}
$$

Now take $i=j$ :

hence $\eta_{i}=(-1)^{\left(1_{2}\right)}$.

$$
\frac{1}{4} \eta_{i} \sum_{k=1}^{16} \eta_{k}=-(-1)^{\left(1_{2}\right)}
$$

Lemma II. Let $\Gamma_{i}$ transform as an irreducible tensor operator of rank $\left(1_{i}\right)$ and let the reduced matrix element of $\Gamma_{i}$ in the four-dimensional irreducible representation be $\left\langle 4\left\|\Gamma_{i}\right\| 4\right\rangle$. Then the following relation holds

$$
\frac{1}{4}\left\langle 4\left\|\Gamma_{i}\right\| 4\right\rangle^{2}=\left(\begin{array}{cc}
1_{i} \\
1 & 1
\end{array}\right)^{*}
$$


Proof. Consider $\sum_{b}\left(\Gamma_{i}\right)_{a b}\left(\Gamma_{i}\right)_{b c}=\eta_{i} \delta_{a c}$, or

$$
\sum_{b}\left\langle 4, a\left|\Gamma_{i}\right| 4, b\right\rangle\left\langle 4, b\left|\Gamma_{i}\right| 4, c\right\rangle=\eta_{i} \delta_{a c} .
$$

We now apply the Wigner-Eckart theorem:

$$
\sum_{b}\left(\begin{array}{lll}
a & 1_{i} & 4 \\
4 & 1 & b
\end{array}\right)\left(\begin{array}{ccc}
b & 1_{i} & 4 \\
4 & 1 & c
\end{array}\right)\left\langle 4\left\|\Gamma^{i}\right\| 4\right\rangle^{2}=\eta_{i} \delta_{a c} .
$$

By using properties of Wigner coefficients we get

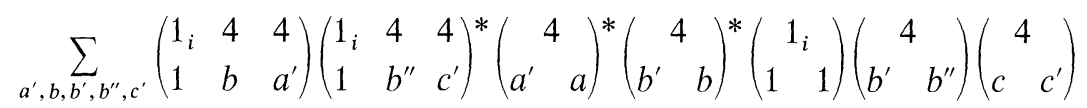

Hence

$$
\times(-1)^{2(4)+\left(1_{i}\right)}\left\langle 4\left\|\Gamma_{i}\right\| 4\right\rangle^{2}=\eta_{i} \delta_{a c} .
$$

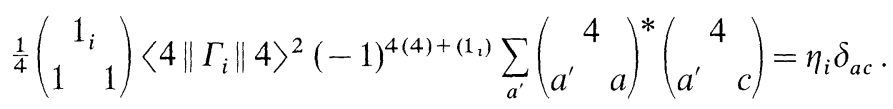

Therefore

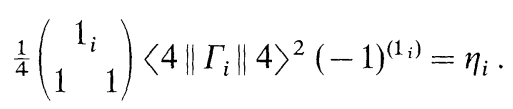

By using Lemma I and multiplying by $\left(\begin{array}{cc}1_{i} \\ 1 & 1\end{array}\right)^{*}$ we find

$$
\frac{1}{4}\left\langle 4\left\|\Gamma_{i}\right\| 4\right\rangle^{2}=\left(\begin{array}{cc}
1_{i} \\
1 & 1
\end{array}\right)^{*} \quad\left(\text { note that }\left(\begin{array}{cc}
1_{i} \\
1 & 1
\end{array}\right)\left(\begin{array}{cc}
1_{i} \\
1 & 1
\end{array}\right)^{*}=1\right) .
$$

We now return to the elementary Fierz transformation. Applying the Wigner-Eckart theorem to formula (15) gives

$$
\begin{aligned}
\left(\begin{array}{lll}
a & 1_{i} & 4 \\
4 & 1 & b
\end{array}\right)\left(\begin{array}{lll}
c & 1_{i} & 4 \\
4 & 1 & d
\end{array}\right)\left\langle 4\left\|\Gamma_{i}\right\| 4\right\rangle^{2} & \\
& =\sum_{k=1}^{16} F_{k i}\left(\begin{array}{lll}
a & 1_{k} & 4 \\
4 & 1 & d
\end{array}\right)\left(\begin{array}{ccc}
c & 1_{k} & 4 \\
4 & 1 & b
\end{array}\right)\left\langle 4\left\|\Gamma_{k}\right\| 4\right\rangle^{2} .
\end{aligned}
$$

Substituting the result of Lemma II provides us with

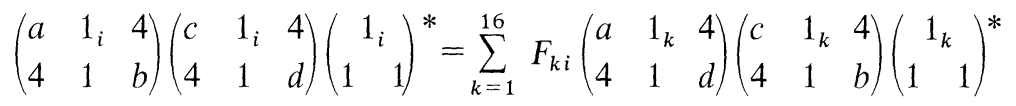

and applying orthogonality relations gives

$$
F_{k i}=\sum_{a, b, c, d}\left(\begin{array}{lll}
a & 1_{i} & 4 \\
4 & 1 & b
\end{array}\right)\left(\begin{array}{lll}
c & 1 & 4 \\
4 & 1_{i} & d
\end{array}\right)\left(\begin{array}{lll}
4 & 1 & d \\
a & 1_{k} & 4
\end{array}\right)\left(\begin{array}{lll}
4 & 1_{k} & b \\
c & 1 & 4
\end{array}\right)
$$


It follows with formula (18) of Ref. [16] that

$$
F_{k i}=-\left\{\begin{array}{ccc}
4 & 4 & 1_{k} \\
4 & 4 & 1_{i}
\end{array}\right\}
$$

Along the same lines it can be shown that in the case of the quaternion group an analogous relationship exists

$$
F_{k i}=-\left\{\begin{array}{ccc}
2 & 2 & 1_{k} \\
2 & 2 & 1_{i}
\end{array}\right\}
$$

\section{d) Derivation of Ordinary Fierz Transformation}

In the previous section we derived for the elementary Fierz transformation the formula

$$
\left(\begin{array}{lll}
a & 1_{i} & 4 \\
4 & 1 & b
\end{array}\right)\left(\begin{array}{lll}
c & 1_{i} & 4 \\
4 & 1 & d
\end{array}\right)\left(\begin{array}{cc}
1_{i} \\
1 & 1
\end{array}\right)^{*}=\sum_{k=1}^{16} F_{k i}\left(\begin{array}{lll}
a & 1_{k} & 4 \\
4 & 1 & d
\end{array}\right)\left(\begin{array}{lll}
c & 1_{k} & 4 \\
4 & 1 & b
\end{array}\right)\left(\begin{array}{cc}
1_{k} \\
1 & 1
\end{array}\right)^{*}
$$

In the case of the ordinary Fierz transformation (cf. Section 3 a) we had to do with sums of this kind of terms, e.g.

$$
\begin{aligned}
\sum_{i \in I}\left(\begin{array}{lll}
a & 1_{i} & 4 \\
4 & 1 & b
\end{array}\right)\left(\begin{array}{lll}
c & 1_{i} & 4 \\
4 & 1 & d
\end{array}\right)\left(\begin{array}{cc}
1_{i} \\
1 & 1
\end{array}\right)^{*} \eta_{i} \\
\quad=\sum_{K} F_{I K} \sum_{k \in K}\left(\begin{array}{lll}
a & 1_{k} & 4 \\
4 & 1 & d
\end{array}\right)\left(\begin{array}{lll}
c & 1_{k} & 4 \\
4 & 1 & b
\end{array}\right)\left(\begin{array}{cc}
1_{k} \\
1 & 1
\end{array}\right)^{*} \eta_{k} .
\end{aligned}
$$

Here $I$ and $K$ were sets of indices. Up to now $I$ and $K$ stood for the usual $S, V, T, A$ and $P$, denoting scalar, vector, tensor, axial vector and pseudo scalar respectively. The symbol $\eta_{i}$ was already defined in Section $3 \mathrm{c}$ with $\left(\Gamma_{i}\right)^{2}=\eta_{i} \cdot 1$. Once again using the orthogonality relation for Wigner coefficients we derive

or

$$
F_{I K}=\sum_{i \in I} \sum_{a, b, c, d}\left(\begin{array}{lll}
a & 1_{i} & 4 \\
4 & 1 & b
\end{array}\right)\left(\begin{array}{lll}
c & 1 & 4 \\
4 & 1_{i} & d
\end{array}\right)\left(\begin{array}{lll}
4 & 1 & d \\
a & 1_{k} & 4
\end{array}\right)\left(\begin{array}{lll}
4 & 1_{k} & b \\
c & 1 & 4
\end{array}\right) \eta_{i} \eta_{k}
$$

$$
F_{I K}=-\sum_{i \in I}\left\{\begin{array}{ccc}
4 & 4 & 1_{i} \\
4 & 4 & 1_{k}
\end{array}\right\} \eta_{i} \eta_{k}, \quad k \in K
$$

As a consequence of lemma I this can also be written as

$$
F_{I K}=-\sum_{i \in I}\left\{\begin{array}{lll}
4 & 4 & 1_{i} \\
4 & 4 & 1_{k}
\end{array}\right\}(-1)^{\left(1_{i}\right)+\left(1_{k}\right)} .
$$

It is to be remarked that it does not matter which $k \in K$ is chosen. Starting with a table for $6 j$-symbols of the Dirac matrix group the $5 \times 5$-Fierz matrix of field theory (see Section 3 a) is recovered. 
We can cast the derived formula for $F_{I K}$ in a much simpler form. To this end we shall introduce an elementary Fierz transformation for upper and lower indices

Hence

$$
\left(\bar{\psi}_{a} \Gamma_{i} \psi_{b}\right)\left(\bar{\psi}_{c} \Gamma^{i} \psi_{d}\right)=\sum_{k} f_{k i}\left(\bar{\psi}_{a} \Gamma_{k} \psi_{d}\right)\left(\bar{\psi}_{c} \Gamma^{k} \psi_{b}\right)
$$

Thus we have

$$
\begin{gathered}
f_{i k}=F_{i k} \eta_{i} \eta_{k} . \\
f_{i k}=\frac{1}{16} \operatorname{Tr}\left(\Gamma_{i} \Gamma_{k} \Gamma_{i} \Gamma_{k}\right) \eta_{i} \eta_{k}=\frac{1}{16} \operatorname{Tr}\left(\Gamma_{i} \Gamma_{k} \Gamma_{i}^{-1} \Gamma_{k}^{-1}\right) \\
=\frac{1}{16} \operatorname{Tr}\left(\chi_{i}^{\left(1_{k}\right)} \Gamma_{k} \Gamma_{k}^{-1}\right) .
\end{gathered}
$$

$$
f_{i k}=\frac{1}{4} \chi_{i}^{\left(1_{k}\right)} .
$$

In the above derivation we made use of the tensorial character of $\Gamma_{k}$

$$
\Gamma_{i} \Gamma_{k} \Gamma_{i}^{-1}=D^{\left(1_{k}\right)}\left(\Gamma_{i}\right) \Gamma_{k}=\chi_{i}^{\left(1_{k}\right)} \Gamma_{k} .
$$

The ordinary Fierz transformation can also be written as

$$
F_{I K}=\sum_{i \in I} f_{i k}=\frac{1}{4} \sum_{i \in I} \chi_{i}^{\left(1_{k}\right)}, \quad k \in K .
$$

\section{e) Some General Properties}

Apart from the Fierz transformation discussed in Sections $3 \mathrm{a}$ and $3 \mathrm{~d}$ we shall show that there are more Fierz-like transformations, which are intermediate with respect to the Lorentz group (see Section 4a). By this we shall mean that it is possible to find sets of indices different from those mentioned in the previous section, such that

$$
F_{I K}=\sum_{i \in I} f_{i k}=\frac{1}{4} \sum_{i \in I} \chi_{i}^{\left(1_{k}\right)}
$$

is independent of the choice of $k \in K$ and furthermore that the matrix $F_{I K}$ possesses all the characteristic properties of the usual Fierz matrix.

Before giving examples of generalized matrices $F_{I K}$ we shall prove some general properties, which hold for the ordinary Fierz matrix as well as for the other ones.

Theorem A. If the sets $I$ and $K$ are chosen such that $F_{I K}=\sum_{i \in I} f_{i k}$, $k \in K$ is independent of the choice of $k$, then

$$
\sum_{K} F_{I K} F_{K J}=\delta_{I J}
$$


Proof. Let

and

$$
F_{I K}=\sum_{i \in I} f_{i k}, \quad k \in K
$$

It follows that

$$
F_{K J}=\sum_{k^{\prime} \in K} f_{k^{\prime} j}, \quad j \in J
$$

$$
\sum_{K} F_{I K} F_{K J}=\sum_{K} \sum_{i \in I} \sum_{k^{\prime} \in K} f_{i k} f_{k^{\prime} j}
$$

Here $k$ has to be taken from the set $K$, but the choice may be different for the various terms in the last summation. Therefore we take in each term $k=k^{\prime}$. Hence

$$
\sum_{K} F_{I K} F_{K J}=\sum_{K} \sum_{i \in I} \sum_{k^{\prime} \in K} f_{i k^{\prime}} f_{k^{\prime} j}=\sum_{i \in I \text { all } k^{\prime}} f_{i k^{\prime}} f_{k^{\prime} j}=\sum_{i \in I} \delta_{i j}=\delta_{I J}
$$

for $\sum_{i \in I} \delta_{i j}=0$ if and only if $I \neq J$.

Therefore we have $\sum_{K} F_{I K} F_{K J}=\delta_{I J}$ or $F^{2}=1$. In this proof the property that $F_{I K}=\sum_{i \in I} f_{i k}$ is independent of the
choice of $k \in K$ is essential.

Of course in order to give a physical meaning to the transformation matrix $F_{I K}$ it is necessary to have $F^{2}=1$.

Theorem B. The Fierz matrix $F_{I K}$ can be symmetrized by rescaling.

This theorem implies that by introducing $\tilde{\Gamma}_{I}=n_{I}^{-\frac{1}{4}} \Gamma_{I}$ instead of $\Gamma_{I}$ and $\tilde{F}_{I K}=\left(n_{I} n_{K}\right)^{-\frac{1}{2}} \sum_{i \in I, k \in K} f_{i k}$ instead of $F_{I K}$ we get

where

$$
\left(\bar{\psi}_{a} \tilde{\Gamma}_{I} \psi_{b}\right)\left(\bar{\psi}_{c} \tilde{\Gamma}^{I} \psi_{d}\right)=\sum_{K} \tilde{F}_{I K}\left(\bar{\psi}_{a} \tilde{\Gamma}_{K} \psi_{d}\right)\left(\bar{\psi}_{c} \tilde{\Gamma}^{K} \psi_{b}\right)
$$

$$
\tilde{F}_{I K}=\tilde{F}_{K I}
$$

Here $n_{I}$ denotes the number of elements of the set $I$.

Corollary. Because of the symmetric (hermitian) character of $\tilde{F}_{I K}$, this matrix has a complete set of eigenvectors, which can be chosen orthogonal. From Theorem $A$ and $B$ it follows that the Fierz matrix $F_{I K}$ has a complete set of eigenvectors with eigenvalues \pm 1 (i.e. invariants and anti-invariants).

Theorem C. Let

and

$$
X_{k}^{\left(1_{j}\right)}=\eta_{k} \chi_{k}^{\left(1_{j}\right)}
$$

$$
X_{K}^{\left(1_{j}\right)}=\sum_{k^{\prime} \in K} X_{k^{\prime}}^{(1, j)}
$$


Then

and

$$
\sum_{k} f_{i k} X_{k}^{\left(1_{j}\right)}=-(-1)^{\left(1_{j}\right)} X_{i}^{\left(1_{j}\right)}
$$

$$
\sum_{K} F_{I K} X_{K}^{\left(1_{j}\right)}=-(-1)^{\left(1_{j}\right)} X_{I}^{\left(1_{j}\right)}
$$

The proofs of Theorems B and C are easy and will be omitted here. Theorem $\mathrm{C}$ is very useful for writing down Fierz invariants and antiinvariants.

\section{Applications}

\section{a) Dirac Matrix Group}

In Section $3 \mathrm{~d}$ we saw already that by choosing $S, V, T, A$ and $P$ for the sets $I$ and $K$ we recovered the ordinary Fierz matrix of formula (5). The Fierz invariants and anti-invariants can be calculated by using Theorem $\mathrm{C}$ and the table of characters of the Dirac matrix group. The invariants are found to be

$$
(1,0,2,0,1) \text { and }(1,2,0,2,-1)
$$

whereas the anti-invariants are

$$
(1,4,-6,-4,1),(1,-2,0,-2,-1) \text { and }(1,-4,-6,4,1) \text {. }
$$

The way in which we found these eigenvectors is much less laborious than the usual procedure of solving the characteristic equation of the $5 \times 5$-Fierz matrix. Of course, because of the degeneracy of the eigenvalues the eigenvectors are not uniquely determined and therefore linear combinations of the above eigenvectors belonging to the same eigenvalue are eigenvectors as well.

As was already mentioned in Section $3 \mathrm{e}$ there are more possible sets of indices $I_{1}, \ldots, I_{n}$ which give rise to a Fierz-like transformation. Because the $\Gamma$-matrices not only play a role in the wave equation invariant under the group $O(3,1)$, but also in the wave equation invariant under $O(4,1)$ (Ref. $[13,17])$ it goes without saying that we should also try the sets

$$
I_{1}=\{1\}, \quad I_{2}=\left\{\gamma^{a}\right\}, \quad I_{3}=\left\{\gamma^{a} \gamma^{b}\right\}
$$

$(a, b=0,1,2,3,5$ and $a<b)$. We call $I_{1}$ the scalar $S, I_{2}$ the vector $V$ and $I_{3}$ the tensor $T$. Indeed it appears that with this choice of the $I_{i} \sum_{i \in I} f_{i k}$
is independent of the element $k \in K$. 
The corresponding Fierz matrix takes the form

(cf. Ref. [8], formula (150)).

$$
F_{I K}=\frac{1}{4}\left[\begin{array}{rrr}
1 & 1 & 1 \\
5 & -3 & 1 \\
10 & 2 & -2
\end{array}\right] \text {. }
$$

There is one invariant

$$
(1,1,2)
$$

and two anti-invariants

$$
(1,5,-10) \text { and }(1,-3,-2) \text {. }
$$

The question arises immediately if we can find still other Fierz transformations. The following sets of $\gamma$-matrices turned out to generate an $8 \times 8$-Fierz matrix

$S=\{1\}, V_{l}=\left\{\gamma^{0}\right\}, V_{t}=\left\{\gamma^{1}, \gamma^{2}, \gamma^{3}\right\}, T_{l}=\left\{\gamma^{0} \gamma^{1}, \gamma^{0} \gamma^{2}, \gamma^{0} \gamma^{3}\right\}$,

$T_{t}=\left\{\gamma^{1} \gamma^{2}, \gamma^{1} \gamma^{3}, \gamma^{2} \gamma^{3}\right\}, A_{l}=\left\{\gamma^{0} \gamma^{5}\right\}, A_{t}=\left\{\gamma^{1} \gamma^{5}, \gamma^{2} \gamma^{5}, \gamma^{3} \gamma^{5}\right\}, P=\left\{\gamma^{5}\right\}$.

The corresponding Fierz matrix is

$$
F_{I K}=\frac{1}{4}\left[\begin{array}{rrrrrrrr}
1 & 1 & 1 & 1 & 1 & 1 & 1 & 1 \\
1 & 1 & -1 & -1 & 1 & -1 & 1 & -1 \\
3 & -3 & -1 & 1 & -1 & 3 & 1 & -3 \\
3 & -3 & 1 & -1 & -1 & -3 & 1 & 3 \\
3 & 3 & -1 & -1 & -1 & 3 & -1 & 3 \\
1 & -1 & 1 & -1 & 1 & 1 & -1 & -1 \\
3 & 3 & 1 & 1 & -1 & -3 & -1 & -3 \\
1 & -1 & -1 & 1 & 1 & -1 & -1 & 1
\end{array}\right] .
$$

The invariants of this generalized Fierz transformation are given by

$$
\begin{gathered}
(1,-1,1,1,1,1,-1,1),(1,1,1,-1,1,1,1,-1) \\
(1,1,-1,1,1,-1,1,1) \text { and }(1,-1,3,3,-3,-1,3,-1)
\end{gathered}
$$

and the anti-invariants by

$$
\begin{aligned}
& (1,1,-3,3,-3,1,-3,-1),(1,1,3,-3,-3,-1,-3,1), \\
& (1,-1,-1,-1,1,-1,-1,-1) \text { and }(1,-1,-3,-3,-3,1,3,1) .
\end{aligned}
$$

We cannot give a quite rigorous justification for the existence of the above mentioned $8 \times 8$-Fierz matrix and a number of other generalized Fierz matrices, to be mentioned below. However, we might interpret the transformation of Eq. (40) by the following reasoning, Let us take an 
arbitrary time-like four-vector $k^{\mu}$. Without loss of generality we can suppose it to have the form $(k, 0,0,0)$. Every Lorentz vector, tensor and axial vector can now be split into a longitudinal and a transverse part with respect to $k^{\mu}$. E.g. for the vector-vector interaction

$$
V=\left(\bar{\psi}_{a} \gamma^{\mu} \psi_{b}\right)\left(\bar{\psi}_{c} \gamma_{\mu} \psi_{d}\right)
$$

we obtain for the longitudinal part

$$
V_{l}=\frac{1}{k^{2}}\left(\bar{\psi}_{a} k \psi_{b}\right)\left(\bar{\psi}_{c} k \psi_{d}\right) .
$$

The transverse part $V_{t}$ is given by $V_{t}=V-V_{l}$.

Similarly we have

$$
T_{l}=\frac{1}{k^{2}}\left(\bar{\psi}_{a} k \gamma^{\mu} \psi_{b}\right)\left(\bar{\psi}_{c} k \gamma_{\mu} \psi_{d}\right)-\left(\bar{\psi}_{a} \psi_{b}\right)\left(\bar{\psi}_{c} \psi_{d}\right)
$$

and

$$
A_{l}=\frac{1}{k^{2}}\left(\bar{\psi}_{a} k \gamma^{5} \psi_{b}\right)\left(\bar{\psi}_{c} k \gamma_{5} \psi_{d}\right)
$$

With the special choice made above we get

$$
\begin{aligned}
V_{l}= & \left(\bar{\psi}_{a} \gamma^{0} \psi_{b}\right)\left(\bar{\psi}_{c} \gamma_{0} \psi_{d}\right), \\
V_{t}= & \left(\bar{\psi}_{a} \gamma^{1} \psi_{b}\right)\left(\bar{\psi}_{c} \gamma_{1} \psi_{d}\right)+\left(\bar{\psi}_{a} \gamma^{2} \psi_{b}\right)\left(\bar{\psi}_{c} \gamma_{2} \psi_{d}\right) \\
& +\left(\bar{\psi}_{a} \gamma^{3} \psi_{b}\right)\left(\bar{\psi}_{c} \gamma_{3} \psi_{d}\right), \\
T_{l}= & \left(\bar{\psi}_{a} \gamma^{0} \gamma^{1} \psi_{b}\right)\left(\bar{\psi}_{c} \gamma_{0} \gamma_{1} \psi_{d}\right)+\left(\bar{\psi}_{a} \gamma^{0} \gamma^{2} \psi_{b}\right)\left(\bar{\psi}_{c} \gamma_{0} \gamma_{2} \psi_{d}\right) \\
& +\left(\bar{\psi}_{a} \gamma^{0} \gamma^{3} \psi_{b}\right)\left(\bar{\psi}_{c} \gamma_{0} \gamma_{3} \psi_{d}\right), \quad \text { etc. }
\end{aligned}
$$

Hence it is understandable that for our special choice of $k^{\mu}, V_{l}$ is represented by $\left\{\gamma^{0}\right\}, V_{t}$ by $\left\{\gamma^{1}, \gamma^{2}, \gamma^{3}\right\}$ etc. as in formula (39). A similar reasoning can be set up when the vector $k^{\mu}$ is space-like, e.g. $(0,0,0, k)$. In this context it is to be noted that in the explicit form of $f_{i k}$ the symbols $\gamma^{0}$, $\gamma^{1}, \gamma^{2}$ and $\gamma^{3}$ play exactly the same role.

In the applications in the beginning of this section we remarked that the groups $O(3,1)$ and $O(4,1)$ were of significance. In this last application it seems that we have to do with the little group belonging to the vector $k^{\mu}$. This little group is isomorphic with the groups $O(2,1)$ or $O(3)$.

As already mentioned still other Fierz transformations can be found by studying the tensors belonging to different subgroups of $O(4,1)$. In this way we find generalized Fierz transformations, which will be listed below together with the transformations already discussed. 


$$
\begin{array}{lll}
\text { Group } & \text { Order } & \text { Index sets } \\
O(4,1) & 3 \times 3 \quad & \{1\},\left\{\gamma^{a}\right\},\left\{\gamma^{a} \gamma^{b}\right\} \\
O(3,1) & 5 \times 5 \quad & \{1\},\left\{\gamma^{\mu}\right\},\left\{\gamma^{\mu} \gamma^{v}\right\},\left\{\gamma^{\mu} \gamma^{5}\right\},\left\{\gamma^{5}\right\} \\
O(2,1) \text { or } O(3) \quad 8 \times 8 \quad & \{1\},\left\{\gamma^{0}\right\},\left\{\gamma^{1}, \gamma^{2}, \gamma^{3}\right\},\left\{\gamma^{0} \gamma^{1}, \gamma^{0} \gamma^{2}, \gamma^{0} \gamma^{3}\right\} \\
& & \left\{\gamma^{1} \gamma^{2}, \gamma^{1} \gamma^{3}, \gamma^{2} \gamma^{3}\right\},\left\{\gamma^{0} \gamma^{5}\right\} \\
& \left\{\gamma^{1} \gamma^{5}, \gamma^{2} \gamma^{5}, \gamma^{3} \gamma^{5}\right\},\left\{\gamma^{5}\right\} \\
& \{1\},\left\{\gamma^{0}, \gamma^{5}\right\},\left\{\gamma^{1}, \gamma^{2}, \gamma^{3}\right\} \\
O(1,1) \otimes O(3) \quad 6 \times 6 & \left\{\gamma^{0} \gamma^{1}, \gamma^{0} \gamma^{2}, \gamma^{0} \gamma^{3}, \gamma^{1} \gamma^{5}, \gamma^{2} \gamma^{5}, \gamma^{3} \gamma^{5}\right\} \\
\text { or } O(2) \otimes O(2,1) & & \left\{\gamma^{1} \gamma^{2}, \gamma^{1} \gamma^{3}, \gamma^{2} \gamma^{3}\right\},\left\{\gamma^{0} \gamma^{5}\right\} \\
O(1,1) \otimes O(2) & 9 \times 9 \quad & \{1\},\left\{\gamma^{0}, \gamma^{1}\right\},\left\{\gamma^{2}, \gamma^{3}\right\},\left\{\gamma^{0} \gamma^{1}\right\} \\
\text { or } O(2) \otimes O(2) \quad & & \left\{\gamma^{0} \gamma^{2}, \gamma^{0} \gamma^{3}, \gamma^{1} \gamma^{2}, \gamma^{1} \gamma^{3}\right\},\left\{\gamma^{2} \gamma^{3}\right\} \\
& \left\{\gamma^{0} \gamma^{5}, \gamma^{1} \gamma^{5}\right\},\left\{\gamma^{2} \gamma^{5}, \gamma^{3} \gamma^{5}\right\},\left\{\gamma^{5}\right\} \\
O(1,1) \text { or } O(2) \quad 12 \times 12 & \{1\},\left\{\gamma^{0}\right\},\left\{\gamma^{1}\right\},\left\{\gamma^{2}, \gamma^{3}\right\},\left\{\gamma^{0} \gamma^{1}\right\} \\
& & \left\{\gamma^{0} \gamma^{2}, \gamma^{0} \gamma^{3}\right\},\left\{\gamma^{1} \gamma^{2}, \gamma^{1} \gamma^{3}\right\},\left\{\gamma^{2} \gamma^{3}\right\},\left\{\gamma^{0} \gamma^{5}\right\}, \\
& \left\{\gamma^{1} \gamma^{5}\right\},\left\{\gamma^{2} \gamma^{5}, \gamma^{3} \gamma^{5}\right\},\left\{\gamma^{5}\right\}
\end{array}
$$

Apart from these transformations still another exists of order $2 \times 2$ apparently not connected with a subgroup of $O(4,1)$ in which the index sets are

$$
\{1\},\left\{\gamma^{a}, \gamma^{a} \gamma^{b}\right\} .
$$

This Fierz matrix is

with the invariant

$$
F=\frac{1}{4}\left[\begin{array}{rr}
1 & 1 \\
15 & -1
\end{array}\right],
$$

$$
(1,3)
$$

and anti-invariant

$$
(1,-5) \text {. }
$$

It is not known to us whether it is possible to interpret this transformation in the same way as the previous ones.

\section{b) Quaternion Group}

Along the same lines a number of Fierz transformations connected with the quaternion group can be obtained. Starting with the elementary Fierz transformation of Section $3 \mathrm{a}$ we find a Fierz transformation of 
order $2 \times 2$ generated by the index sets $\left\{\Sigma_{1}\right\},\left\{\Sigma_{2}, \Sigma_{3}, \Sigma_{4}\right\}$ :

$$
F=\frac{1}{2}\left[\begin{array}{rr}
1 & 1 \\
3 & -1
\end{array}\right]
$$

with invariant

$$
(1,1)
$$

and anti-invariant

$$
(1,-3)
$$

Furthermore the index sets $\left\{\Sigma_{1}\right\},\left\{\Sigma_{2}\right\},\left\{\Sigma_{3}, \Sigma_{4}\right\}$ give

$$
F=\frac{1}{2}\left[\begin{array}{rrr}
1 & 1 & 1 \\
1 & 1 & -1 \\
2 & -2 & 0
\end{array}\right],
$$

with invariants

and anti-invariant

$$
(1,-1,2) \text { and }(1,1,0)
$$

$$
(1,-1,-2) \text {. }
$$

The first of these transformations has to do with $O(3)$. In essence the same transformation is given by formulae (154a) and (154b) of Ref. [8] and formulae $(28,16)$ and $(28,17)$ of Ref. [18].

The second transformation is connected with the little group $O(2)$.

The authors are indebted to Professor Dr. H. J. Groenewold for many discussions and to Professor Dr. A. F. Yano for reading the manuscript. One of us (E. de V.) has carried out this work as a scientific staff member of the Stichting F.O.M. (Foundation for Fundamental Research of Matter) which is financially supported by the Netherlands Organization for Pure Scientific Research (Z.W.O.).

\section{References}

1. Fierz, M.: Z. Physik 102, 572 (1936).

2. Umezawa, H.: Quantum field theory, p. 118. Amsterdam: North-Holland Publ. Comp. 1956.

3. Good, R. H., Jr.: Rev. Mod. Phys. 27, 210 (1955).

4. Nambu, Y., Jona-Lasinio, G.: Phys. Rev. 122, 345 (1960).

5. Lurié, D., Macfarlane, A. J. : Phys. Rev. 136 B, 816 (1964).

6. De Graaf, T., Tolhoek, H. A.: Nucl. Phys. 81, 596 (1966).

7. Feldman, G., Fulton, T., Matthews, P. T.: Nuovo Cimento 50 A, 349 (1967).

8. Case, K. M.: Phys. Rev. 97, 810 (1955).

9. Barut, A. O., Unal, B. C.: Nuovo Cimento 28, 112 (1963).

10. Foldy, L. L., Peierls, R. F.: Phys. Rev. 130, 1585 (1963).

11. Carruthers, P. A., Krisch, J. P.: Ann. Phys. (N. Y.) 33, 1 (1965).

12. Lee, H.: J. Math. Phys. 10, 779 (1969). 
13. Boerner, H.: Darstellungen von Gruppen (zweite Auflage). Berlin-Heidelberg-New York: Springer 1967.

14. Freudenthal, H.: Proc. Kon. Ned. Ak. v. Wet., Ser. A, 59, 515 (1956) (== Indag. Math. 18, 515 (1956)).

15. Hamermesh, M.: Group theory and its applications to physical problems. Reading Mass.: Addison-Wesley Publ. Comp. 1962.

16. Wigner, E. P.: On the matrices which reduce the Kronecker product of representations of S.R. groups (Princeton, 1951), published in: Biedenharn, L. C., Van Dam, H.: Quantum theory of angular momentum. New York: Academic Press 1965.

17. De Vos, J. A., Hilgevoord, J.: Nucl. Phys. B1, 494 (1967).

18. Beresteckij, V. B., Lifšıc, E. M., Pitaevskij, L. P.: Reljativistskaja kvantovaja teorija (čast' 1). Moskva: Nauka 1968.

\author{
E. de Vries \\ Institute for Theoretical Physics \\ University of Groningen \\ Groningen, Holland
}

